\title{
G

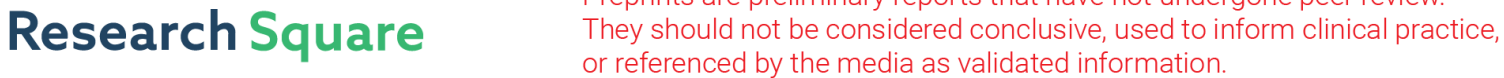

\section{Prognostic Value of Olfactory and Gustatory Dysfunction As Initial Symptoms of COVID-19 Infection}

\section{Ameen Biadsee ( $\sim$ ameenbiadsee@gmail.com )}

Department of Otorhinolaryngology - Head and Neck Surgery, Meir Medical Center, Kfar-Saba, Israel https://orcid.org/0000-0002-3768-6216

\section{Or Dagan}

Department of Otorhinolaryngology - Head and Neck Surgery, Hillel Yaffe Medical Center, Hadera, Israel

\section{Zeev Ormianer}

Department of Oral Rehabilitation, The Maurice and Gabriela Goldschleger School of Dental Medicine,

Tel Aviv University, Tel Aviv, Israel

\section{Firas Kassem}

Department of Otorhinolaryngology - Head and Neck Surgery, Meir Medical Center, Kfar-Saba, Israel

\section{Shchada Masarwa}

Department of Oral Rehabilitation, The Maurice and Gabriela Goldschleger School of Dental Medicine,

Tel Aviv University, Tel Aviv, Israel

\section{Ameer Biadsee}

Department of Oral Rehabilitation, The Maurice and Gabriela Goldschleger School of Dental Medicine,

Tel Aviv University, Tel Aviv, Israel

\section{Research Article}

Keywords: COVID-19 recovery, smell dysfunction, gustatory dysfunction, post-viral olfactory function loss

Posted Date: October 6th, 2020

DOI: https://doi.org/10.21203/rs.3.rs-85423/v1

License: (c) (i) This work is licensed under a Creative Commons Attribution 4.0 International License.

Read Full License 


\section{Abstract}

Introduction: Olfactory and gustatory chemosensory dysfunctions are very prevalent in COVID-19 infection, our aim is to further explore chemosensory recovery among these patients.

Methods: This is a cross-sectional follow-up study, which serves as a sequel to a study by Biadsee, et al. Among the previous study population of 128 non-hospitalized patients, who were tested positive for COVID-19 by reverse transcription-polymerase chain reaction (RT-PCR). Ninety-seven patients participated in a telephone survey designed for this study. Recovery of olfactory and gustatory functions were defined when a score is greater or equal to that reported before the disease. Persistent hyposmia and hypogeusia were defined as lower scores reported during follow-up, compared with scores before the disease.

Results: A total of 43 men and 54 women were included. Mean age was 37 years (range 18-73). Mean follow-up was 59.2 days (range 21-83). At follow-up, 72 (74.2\%) patients reported normalization of gustatory function and $63(64.9 \%)$ reported normalization of olfactory function. No patient reported nasal obstruction at follow-up. Recovery of olfactory function was positively correlated with gustatory recovery $(p=0.003)$. Twenty-three $(23.7 \%)$ reported persistent xerostomia. Women who reported persistent xerostomia had higher rates of olfactory and gustatory dysfunction ( $p=0.006$ and $p=0.008$, respectively) than those who did not. Olfactory and gustatory disorders as initial symptoms were negative prognostic factors for recovery.

Conclusion: Olfactory and/or gustatory dysfunction as a presenting symptom of COVID-19 infection may be a negative prognostic factor for full recovery of olfactory and gustatory function.

\section{Introduction}

According to recently published data, olfactory and gustatory chemosensory impairment are very prevalent in COVID-19 infection. In our previous study(1), we have reported initial symptoms and prevalence of olfactory and gustatory dysfunction among COVID-19 patients. We found that impaired sense of smell or taste as a presenting symptom can be as high as $38 \%$. Similar to our results, Coelho et al.(2) found that $37.7 \%$ of their study group reported subjective olfactory or gustatory dysfunction (OD and GD, respectively) as a presenting symptom. Recovery rates of chemosensory impairment are being investigated, worldwide. Few studies have tracked outcomes at serial time points and have shown a gradual improvement in olfactory and gustatory functions over time. $(3,4)$ Further analysis by Paderno et al.(4) found nasal congestion, gender, and grade of dysfunction, as risk-factors for late resolution from OD. However, there are no data regarding recovery patterns of patients who reported OD and GD as initial symptoms. The aim of this study was to investigate recovery rates of OD and GD after COVID-19 infection.

\section{Methods}


This study was conducted in the Department of Oral Rehabilitation, School of Dental Medicine of Tel Aviv University, in collaboration with the Otorhinolaryngology Department, Meir Medical Center (affiliated with Tel Aviv University).

This is a sequel to the previous study by Biadsee et al.(1) It was approved by the Tel Aviv University Ethics Committee (application number 0001623-2). Informed consent was obtained from all participants, by telephone.

\section{Participants}

Of the previous study population of 128 non-hospitalized patients, who were tested positively for COVID19 by reverse transcription-polymerase chain reaction (RT-PCR) and considered to have mild symptoms. Ninety-seven patients (75.8\%) agreed to participate in the follow-up telephone survey, 21 refused and 10 could not be reached. All patients had recovered from COVID-19 disease based on 2 negative reverse transcription-polymerase chain reaction (RT-PCR) assay results, on sequential samples taken at least 24 hours apart.

\section{The Survey}

A 7-question telephone questionnaire was designed for this study, which was conducted on June 15, 2020 (Table 1). The first section contained questions regarding the date of second negative RT-PCR test and the last symptom at the time of disease. The period of illness was defined as the number of days between the initial positive detection date by PCR (obtained from the previous study(1)) and the date of the second negative PCR test. The follow-up period was defined as the number of days between the second negative PCR test and the date of the survey (Figure 1).

Section 2 included a single dichotomous question regarding current feeling of xerostomia.

In Sections 3 and 4, subjective smell and taste functions were each measured using a 10-point numerical scale from 0 to 10, with 0 representing anosmia and ageusia and 10 representing a very good sense of smell and taste, respectively.

Scores of olfactory and gustatory functions of the pre-ailment and during the disease were obtained from the previous study data, scores were not revealed to the patient at the time of the current survey. Normalization of olfactory and gustatory functions were defined as a score greater than or equal to the scores reported before the disease; obtained from the previous study data.(1). OD and GD were defined as a score lower than reported scores before the disease.

Information obtained from the questionnaires was tallied and summarized.

\section{Statistical Analysis}

Statistical data was analyzed using the R Project for Statistical Computing, version 3.6.2. Reported measures were tested for the association with reported initial loss of smell taste (yes\no), with 
consideration of the phase of reporting. The prognosis was fitted in correspondence using repeated measures linear regression.

Statistical significance of each covariate was tested using the likelihood ratio test.

Post-hoc analysis was later performed to test for hypotheses of interest. Namely, comparing olfactory and taste recovery across the three phases (Before, during and post), either within groups by initial loss of smell $\backslash$ taste (yes $\backslash$ no), and for all patients combined. All tests and credible intervals are reported at a level of $\mathrm{a}=5 \%$.

\section{Results}

A total of 43 men and 54 women were included in the study. Mean age was 37 years (range $18-73$ ). Mean follow-up period was 59.2 days (range $21-83$ ). Mean duration of illness from diagnosis to second PCR test was 26.2 days (range $5-58$; Table 2 ).

\section{Taste}

Thirty-four (35.1\%) patients reported taste dysfunction as an initial symptom of COVID-19. Mean scores of taste function were; 9.4 before the disease, 5.8 during the ailment period and 8.8 at follow-up. There was no significant statistical difference between the sexes ( $p=0.530$ before, $p=0.181$ during and $p=0.945$ after (Table 3). However, patients who reported gustatory dysfunction as an initial symptom had significantly lower scores at follow-up period comparing to those patients who did not report GD as an initial symptom (mean scone $8.6 \mathrm{vs}$. 9, respectively, $p=0.003$ ). Length of the aliment period did not affect the normalization of gustatory function $(p=0.420)$.

Seventy-two patients $(74.2 \%)$ reported normalization of gustatory function $(p=0.018)$. While $25(25.8 \%)$ reported persistent dysfunction at the time of the survey ( 9 men and 16 women) with no significant difference between the sexes $(p=0.330)$. Gustatory or olfactory dysfunction as an initial symptom was a negative prognostic factor for recovery of gustatory function ( $p=0.03$ and $p=0.04$, respectively).

\section{Smell}

Thirty-eight (39.2\%) patients reported olfactory dysfunction as an initial symptom of COVID-19 disease. Mean scores of smell function were; 9.1 before the disease, 5 in the ailment period and 8.4 at the time of follow-up. There were no significant statistical differences between the sexes in terms of disease phase $(p=0.580,0.060$ and $p=0.083$, respectively; Table 3 ). However, patients who reported olfactory dysfunction as an initial symptom had significantly lower scores at follow-up period comparing to those patients who did not report OD as an initial symptom (mean scone 7.9 vs. 8.7, respectively, $p<0.001$ ). None of the patients reported nasal congestion or rhinorrhea during the follow-up period.

Sixty-three patients (64.9\%) reported normalization of olfactory function $(p=0.008)$, while $33(34 \%), 12$ men and 22 women, reported partial recovery of smell function by the time of the survey. There was no 
significant difference between the sexes $(p=0.188)$. One patient reported continued anosmia during the follow-up period.

A longer disease period did not influence the rate of olfactory function recovery $(p=0.933)$. However, olfactory or gustatory dysfunction as a presenting symptom of COVID-19 was a negative prognostic factor for recovery of olfactory function $(p=0.000$ and $p=0.007$, respectively).

Recovery of olfactory function was positively associated with gustatory recovery for both men and women $(p=0.003)$.

\section{Dry mouth}

Sixty patients (61.9\%) reported xerostomia during the ailment period and $23(23.7 \%)$ reported persistent xerostomia at the time of the survey; 9 men and 14 women, with no significant difference between the $\operatorname{sexes}(p=0.566)$.

Women who reported persistent xerostomia had a higher rate of olfactory and gustatory dysfunction ( $p=0.006$ and $p=0.008$, respectively) than those who did not.

Patients who reported persistent xerostomia had a lower rates of gustatory function recovery. $(p=0.005)$

Seven patients reported persistent myalgia and cough.

\section{Discussion}

The current study investigated the recovery rates of olfactory and gustatory dysfunction among recovered COVID-19 patients who were not hospitalized while infected.

Smell and gustatory dysfunction are very prevalent in COVID-19 disease. In our previous cohort(1), we reported olfactory and gustatory dysfunction rates as high as $67 \%$ and $52 \%$, respectively. Complete recovery of smell function was found in $64.9 \%$ of patients, while $34 \%$ reported only partial recovery. As reported by Chary et al.(5), we also found no significant difference between the sexes concerning recovery of smell function. However, Paderno et al.(4) found that later recovery of smell was more prevalent among women. This may be because women are more sensitive to minor smell alteration.(6)

The oral cavity strongly expresses angiotensin-converting enzyme 2 (ACE II), particularly in the tongue.(7) A recent study demonstrated that ACE II inhibitors could engender loss of taste perception by alterations in the delicate mechanisms involving G-protein coupled proteins and sodium channels.(8) Moreover, another report suggested that ACE II receptors play a crucial role in cellular entry and are a potential cause of COVID-19 infection.(9) This can explain why dysgeusia is very prevalent in COVID-19 patients. We found a high rate of gustatory recovery (74.2\%) among the study group, which can be explained by the regenerative ability of chemosensory receptor cells in the oral cavity.(10) 
Olfactory and/or gustatory dysfunction have been widely reported as one of the sole initial manifestations of COVID-19 infection. $(1,4,11)$ In our cohort, we found that patients who presented with $\mathrm{OD}$ and/or GD as an initial symptom had significantly lower rates of complete recovery of olfactory and gustatory functions $(p=0.000$ and $p=0.007$, respectively).

To the best of our knowledge, no current study in the literature has reported a relation between OD and GD as initial symptoms and different chemosensory recovery rates.

In this analysis, which is a follow-up to the previous study by Biadsee et al.(1), we aimed to further understand the long term impact of the damage to the olfactory neuroepithelium caused by the COVID-19 virus. Chary et al.(5) found that 70\% of COVID-19 patients reported OD without nasal obstruction. Furthermore, $64 \%$ of their cohort had complete resolution of OD within 15 days after positive PCR diagnosis. D'Ascanio et al.(3) and Paderno et al.(4) found that OD was completely resolved for $84.3 \%$ and $88 \%$, respectively, 30 days after diagnosis. Lechien et al.(12) reported complete resolution of OD among $72.6 \%$ of patients, within 8 days after the resolution of the disease. Xiong et al.(13) found $11 \%$ of their study group had residual OD 2-4 weeks after negative PCR.

None of the abovementioned studies reported on concomitant nasal obstruction rates during follow-up. In our follow-up period, a mean of 59.2 days after the second negative PCR test, we found that OD had resolved in $64.9 \%$ of the patients and none reported residual nasal obstruction. Our analysis is characterized by a relatively long follow-up period and high percentage of residual OD compared to reports in the literature $(3,4,12,14-16)$ and provides a complementary data regarding the clinical course of $\mathrm{OD}$ and GD.

Post-viral olfactory loss is defined as the persistence or appearance of olfactory dysfunction after recovery from upper respiratory infection.(17) Suggested mechanisms for post-viral olfactory loss include the ability of viruses to penetrate the brain via the fovea ethmoidalis and elicit an immune response that can lead to neuroepithelial damage.(18) The relatively long follow-up period and high percentage of residual OD compared to reports in the literature may suggest that post-viral olfactory loss was more common among our cohort of patients.

Similar to our previous study results, we found that persistent xerostomia was significantly related to hypogeusia. Hypogeusia may cause reduced salivary production since taste is considered the major stimulant of saliva formation.

The main limitations of this study are its relatively small size and the lack of objective measurements for diagnosis of OD and GD. As a follow up study on the same participates, there is a possibility of recall bias. In order to minimize the risk of recall bias we used two strategies; the first, previous scores of each patient were not revealed to him during the present telephone survey. Secondly, the substantial time that elapsed between the two surveys would serve as a "wash-out period". 
Nevertheless, the availability of references in the literature, helped in interpreting the results. Another strength is the long follow-up (a mean of 59.2 days after the second negative PCR test), which enabled good interpretation of patients' outcomes of 2 months after recovery.

\section{Conclusion}

$\mathrm{OD}$ and GD as a presenting symptom may be a negative prognostic factor for full recovery of olfactory and gustatory function. Further studies are needed to correlate objective olfactory testing with survey data to validate the impact of $O D$ and $G D$ as a presenting symptom on recovery rates.

\section{References}

1. Biadsee A, Biadsee A, Kassem F, Dagan O, Masarwa S, Ormianer Z. Olfactory and Oral Manifestations of COVID-19: Sex-Related Symptoms-A Potential Pathway to Early Diagnosis. Otolaryngol Head Neck Surg. 2020 Jun 16;194599820934380.

2. Coelho DH, Kons ZA, Costanzo RM, Reiter ER. Subjective Changes in Smell and Taste During the COVID-19 Pandemic: A National Survey-Preliminary Results. Otolaryngol Head Neck Surg. 2020 May 19;163(2):302-306.

3. D'Ascanio L, Pandolfini M, Cingolani C, Latini G, Gradoni P, Capalbo M, et al. Olfactory Dysfunction in COVID-19 Patients: Prevalence and Prognosis for Recovering Sense of Smell. Otolaryngol Head Neck Surg. 2020 Jul 14;194599820943530.

4. Paderno A, Mattavelli D, Rampinelli V, Grammatica A, Raffetti E, Tomasoni M, et al. Olfactory and Gustatory Outcomes in COVID-19: A Prospective Evaluation in Nonhospitalized Subjects. Otolaryngol Head Neck Surg. 2020 Jun 30;194599820939538.

5. Chary E, Carsuzaa F, Trijolet J-P, Capitaine A-L, Roncato-Saberan M, Fouet K, et al. Prevalence and Recovery From Olfactory and Gustatory Dysfunctions in Covid-19 Infection: A Prospective Multicenter Study. Am J Rhinol Allergy. 2020 Jun 12;1945892420930954.

6. Frasnelli J, Hummel T. Olfactory dysfunction and daily life. Eur Arch Otorhinolaryngol. 2005 Mar;262(3):231-235.

7. Xu H, Zhong L, Deng J, Peng J, Dan H, Zeng X, et al. High expression of ACE2 receptor of 2019-nCoV on the epithelial cells of oral mucosa. Int J Oral Sci. 2020 Feb 24;12(1):8.

8. Tsuruoka S, Wakaumi M, Araki N, loka T, Sugimoto K, Fujimura A. Comparative study of taste disturbance by losartan and perindopril in healthy volunteers. J Clin Pharmacol. 2005 Nov;45(11):1319-1323.

9. Zhou P, Yang X-L, Wang X-G, Hu B, Zhang L, Zhang W, et al. A pneumonia outbreak associated with a new coronavirus of probable bat origin. Nature. 2020 Feb 3;579(7798):270-273.

10. Oakley B, Riddle DR. Receptor cell regeneration and connectivity in olfaction and taste. Exp Neurol. 1992 Jan;115(1):50-54. 
11. Kaye R, Chang CWD, Kazahaya K, Brereton J, Denneny JC. COVID-19 Anosmia Reporting Tool: Initial Findings. Otolaryngol Head Neck Surg. 2020 Apr 28;163(1):132-134.

12. Lechien JR, Chiesa-Estomba CM, De Siati DR, Horoi M, Le Bon SD, Rodriguez A, et al. Olfactory and gustatory dysfunctions as a clinical presentation of mild-to-moderate forms of the coronavirus disease (COVID-19): a multicenter European study. Eur Arch Otorhinolaryngol. 2020 Aug;277(8):2251-2261.

13. Li J, Long X, Zhu C, Wang H, Wang T, Lin Z, et al. Olfactory Dysfunction in Recovered Coronavirus Disease 2019 (COVID-19) Patients. Mov Disord. 2020 Jun 30;35(7):1100-1101.

14. Lee Y, Min P, Lee S, Kim SW. Prevalence and Duration of Acute Loss of Smell or Taste in COVID-19 Patients. J Korean Med Sci. 2020 May 11;35(18):e174.

15. Hopkins C, Surda P, Whitehead E, Kumar BN. Early recovery following new onset anosmia during the COVID-19 pandemic - an observational cohort study. J Otolaryngol Head Neck Surg. 2020 May 4;49(1):26.

16. Paderno A, Schreiber A, Grammatica A, Raffetti E, Tomasoni M, Gualtieri T, et al. Smell and taste alterations in COVID-19: a cross-sectional analysis of different cohorts. Int Forum Allergy Rhinol. 2020 May 14;

17. Seiden AM. Postviral olfactory loss. Otolaryngol Clin North Am. 2004 Dec;37(6):1159-1166.

18. Baker H, Beth Genter M. The Olfactory System and the Nasal Mucosa as Portals of Entry of Viruses, Drugs, and Other Exogenous Agents into the Brain. In: Doty R, editor. Handbook of olfaction and gustation. CRC Press; 2003.

\section{Tables}

Table 1. Study questionnaire

Section 1.

1. Date of second negative RT-PCR test:

2. What was the last symptom you had and when was the last time you felt it:

Section 2.

3. Do you still feel the need to drink more than usual (dry mouth)? Yes/No

Section 3.

4. Do you feel a change in your sense of taste? Yes/No

5. Rate your sense of taste to date, on a scale from 0 to 10

Section 4 .

6. Do you feel a change in your sense of smell? Yes/No

7. Rate your sense of smell to date, on a scale from 0 to 10 
Table 2. Patient demographics

\begin{tabular}{|l|c|c|c|}
\hline Variable & Men & Women & Total \\
\hline Mean age (year \pm SD) & $38.5 \pm 15.9$ & $35.7 \pm 17.8$ & $37 \pm 17$ \\
\hline Ailment period (days \pm SD) & $26 \pm 10.5$ & $26.3 \pm 9.7$ & $26.2 \pm 10$ \\
\hline Fellow-up period (days \pm SD) & $59.5 \pm 11.2$ & $59 \pm 10.7$ & $59.2 \pm 10.8$ \\
\hline Initial loss of taste symptom, $\mathrm{n}(\%)$ & $13(13)$ & $21(21)$ & $34(35)$ \\
\hline Initial loss of smell symptom ${ }^{\mathrm{a}}, \mathrm{n}(\%)$ & $17(17)$ & $21(21)$ & $38(39)$ \\
\hline
\end{tabular}

a Data obtained from the previous study.(1)

Table 3. A comparison of taste and smell scores between males and females.

\begin{tabular}{ccc|cc}
\hline & \multicolumn{2}{c|}{ Mean Taste score } & \multicolumn{2}{c}{ Mean Smell score } \\
\cline { 2 - 5 } Male & Before $^{\mathrm{a}}$ & After & Before $^{\mathrm{a}}$ & After \\
Female & $9.37 \pm 1$ & $8.88 \pm 2.3$ & $9.05 \pm 1.4$ & $8.91 \pm 1.9$ \\
$p$ value & $9.5 \pm 0.9$ & $8.85 \pm 2.1$ & $9.2 \pm 1.2$ & $8.15 \pm 2.2$ \\
\hline \hline
\end{tabular}

a Scores obtained from pervious study(1).

Figures 


\section{Detection date}

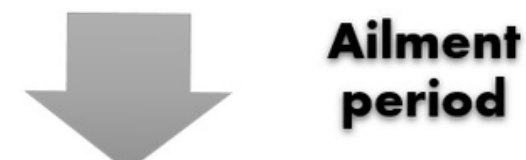

\section{Second}

negative PCR

test date

Follow-up

period

\section{Survey date}

\section{Figure 1}

Study time-line from initial COVID-19 diagnosis to survey (June 15, 2020). Detection date; date of positive PCR test for COVID-19. Survey date; June 15,2020. Ailment period; number of days between detection date and second negative PCR test for COVID-19. Follow-up period; number of date between second negative PCR date and survey date. 